\title{
Percepção de expressões faciais em pessoas com esquizofrenia: movimentos oculares, sintomatologia e nível intelectual
}

\author{
Katerina Lukasova \\ Elizeu Coutinho de Macedo 1 \\ Marcel Chucre Valois \\ Gamaliel Coutinho de Macedo \\ José Salomão Schwartzman
}

\begin{abstract}
Resumo
A análise dos padrões dos movimentos oculares em esquizofrênicos mostra que estes apresentam alterações próprias da doença. O objetivo foi avaliar e relacionar as propriedades dos movimentos oculares com o estado clínico e nível intelectual durante observação de faces. Foram avaliados 10 sujeitos com diagnóstico de esquizofrenia e 10 controle, pareados em função de sexo, idade e escolaridade. Foi utilizada a Escala das Síndromes Positiva e Negativa (ESPN), o Teste de Matrizes Progressivas Raven e o Penn Emotion Acuity Test. A busca visual foi registrada com programa EyeGaze ${ }^{\circledR}$. Resultados mostram que ambos os grupos fixaram mais as faces com conteúdo emocional, sendo o número total de fixações menor entre os pacientes com esquizofrenia. A duração da fixação teve correlação inversa com a pontuação no Raven, PANSS e número de fixação. Parâmetros dos movimentos oculares se correlacionaram com a condição clínica e nível intelectual dos pacientes com esquizofrenia. Palavras-chave: Esquizofrenia; Movimento ocular; Inteligência; Estado clínico.
\end{abstract}

\section{The perception of facial expressions in people with schizophrenia: Eye movements, symptomatology and intelligence}

\begin{abstract}
Analyses of the eye movement patterns of schizophrenics show some specific characteristics underlying the illness. The objective of present study was to evaluate and correlate basic properties of the eye movements with the clinical state and intelligence during the visual scan of faces. 10 outpatient subjects with schizophrenia and 10 controls matched in gender, age and school years were evaluated. The assessment tools were the Positive and Negative Syndrome Scale (PNSS), the Raven's Progressive Matrices Test and the Penn Emotion Acuity Test. The visual scan was registered with the EyeGaze ${ }^{\circledR}$ program. Both groups showed more fixations for stimuli with emotional expression, with significantly smaller overall number of fixations for the schizophrenic subjects group. Duration of fixations was inversely correlated with score in the Raven Test, PANSS and number of fixations. The properties of the eye movements showed correlation with the clinical condition and intelligence of the outpatients with schizophrenia.

Keywords: Schizophrenia; Eye movements; Intelligence; Clinical state.
\end{abstract}

\section{Introdução}

A esquizofrenia é uma doença que atinge cerca de $1 \%$ de população mundial (OMS, 2000). Dados do Ministério de Saúde indicam que de $0,7 \%$ a $1 \%$ da população brasileira já sofreu um surto de esquizofrenia. Os gastos anuais do Sistema Único de Saúde (SUS) com a internação e tratamento de 131 mil pacientes com esquizofrenia consomem quantias elevadas de recursos (Javitt e Coyle, 2004). Os altos custos de internação e tratamento poderiam ser diminuídos se existissem instrumentos de diagnóstico para detecção precoce de pessoas com risco, principalmente entre os familiares de portadores de esquizofrenia.

Estudos têm sido conduzidos a fim de identificar diferentes tipos de alterações fisiológicas e cognitivas presentes antes e durante a erupção da doença. Essas alterações, chamadas de marcadores biológicos, refletem as propriedades hereditárias da doença e em grande parte são encontradas também nos familiares de primeiro grau (Calkins, Iacono \& Curtis, 2003; Ettinger e cols., 2004;

\footnotetext{
${ }^{1}$ Endereço para correspondência:

Programa de Pós-Graduação em Distúrbios do Desenvolvimento

Rua da Consolação, 896 - Consolação - 01302-907 - São Paulo-SP - Tel: (11) 3236-8878

E-mail: elizeumacedo@uol.com.br
} 
Holzman, Proctor \& Huges, 1988). Identificação dos marcadores biológicos específicos da esquizofrenia permitirá a criação de novos instrumentos para diagnóstico diferencial precoce e prevenção. Entre os marcadores, destaca-se a análise do padrão de movimento ocular em pessoas com esquizofrenia. A importância desse marcador foi reconhecida pela $15^{a}$ Conferência de Pesquisadores da Organização Mundial de Saúde (OMS), que propôs e posteriormente executou estudo internacional cujos resultados mostraram que é possível discriminar, com sensibilidade e especificidade de cerca de $90,0 \%$, os pacientes esquizofrênicos das outras doenças psiquiátricas e dos sujeitos normais analisando os movimentos oculares (Kojima e cols., 2001).

As propriedades básicas do movimento ocular são: fixações e movimentos sacádicos. A fixação ocorre quando o olho pára durante um breve período de tempo $(200$ - 300 $\mathrm{ms}$ ) para permitir que o ponto fixado da imagem visual seja focado pela fóvea e processado pelo sistema visual (Rayner, 1998). Os rápidos movimentos dos olhos entre as fixações são conhecidos como sacadas. $O$ comprimento desses movimentos depende do estímulo observado, sendo maior quando o estímulo é uma imagem e menor quando é texto. No entanto, a sensibilidade do sistema visual para as imagens captadas durante esses movimentos é baixa em razão do efeito de supressão sacádica.

Estudos que analisam os movimentos oculares têm mostrado diferenças entre pacientes com esquizofrenia e controles tanto para a duração e localização das fixações quanto para os movimentos sacádicos. Tais diferenças têm sido observadas em tarefas de seguimento contínuo (Holzman, Proctor \& Huges, 1973), movimentos de olhos fechados (Nakamura e cols., 2003) e movimentos oculares exploratórios (Hori e cols., 2002; Matsushima e cols., 1998; Obayashi e cols., 2003).

Os movimentos oculares têm sido estudados com a apresentação de diferentes estímulos. Estudos que analisam o padrão de exploração visual de faces humanas em pessoas com esquizofrenia procuram compreender o porquê de pessoas com esquizofrenia apresentarem contato social empobrecido e rebaixamento na capacidade de reconhecer as emoções pelas expressões faciais. Segundo Goldman e cols. (1993), a dificuldade dos esquizofrênicos de discriminar as emoções pela expressão facial está relacionada com a prevalência de sintomas negativos e com o curso da doença. Os sintomas negativos, tais como embotamento afetivo, falta de vontade e apatia, diminuem o contato social do paciente e, por conseqüência, levam a menor necessidade ou experiência em interpretar as emoções sutis nas faces dos outros. Ao mesmo tempo, a expressividade facial dos pacientes com prevalência dos sintomas negativos está rebaixada, prejudicando a interpretação dos seus estados emocionais pelos outros.
Loughland, Williams e Gordon (2002) observaram o comportamento visual de pacientes esquizofrênicos, com transtorno afetivo e sem histórico psiquiátrico durante a observação de faces humanas classificadas como felizes, tristes e neutras. Resultados mostraram que as estratégias que os esquizofrênicos empregaram para a exploração visual eram limitadas, sendo observado: baixo número de fixações, menor distância entre elas e maior concentração central. Embora os resultados não tenham sido influenciados pelo conteúdo afetivo do estímulo, foi observada uma tendência nos esquizofrênicos a não focar diretamente os traços dominantes do rosto (olhos, nariz e boca), com a exceção da face triste versus neutra, que seguiu o padrão dos sujeitos normais.

Loughland e colaboradores (2004) reaplicaram o mesmo estudo em pacientes com esquizofrenia, seus parentes de primeiro grau e controles. O padrão de busca visual em pacientes foi similar com estudo anterior, com estratégia de exploração visual limitada. Os parentes de primeiro grau exibiram o padrão de exploração visual parecido com os controles, porém nas faces tristes versus felizes mostraram uma surpreendente tendência a não focar traços dominantes. Já os esquizofrênicos e controles mostraram uma tendência, observada também no estudo anterior, de focar traços dominantes em faces tristes. Tais resultados sugeriram a possibilidade de que a falta de atenção para os traços faciais dominantes pode estar presente antes da erupção da doença.

Até que nível o julgamento das emoções é prejudicado pela menor exploração visual dos traços faciais dominantes? Kohler, Bilker, Haagendoorn \& Gur (2000) realizaram estudo com julgamento de expressão facial em 5 diferentes tipos de emoções (triste, feliz, medo, nojo, bravo e neutro) com a graduação de intensidade para cada estado. Entre os pacientes com esquizofrenia foi observada maior dificuldade de diferenciar as intensidades de expressão emocional menor versus extrema. Análise de erros mostrou, que os esquizofrênicos tendem a confundir sinais de expressão facial neutra, atribuindo-lhes emoções negativas. De acordo com Loughland e colaboradores (2004), entre as expressões de emoções, as neutras são as que mais se assemelham às expressões negativas e a diferença entre ambas é sutil.

Sachs e colaboradores (2004) estudaram o julgamento dos pacientes sobre a intensidade das emoções. Resultados mostraram que os esquizofrênicos falham em perceber diferenças sutis no julgamento da intensidade de emoções, embora a discriminação das principais esteja preservada.

A dificuldade no reconhecimento das emoções sutis deve-se, de acordo com Williams, Loughland, Gordon \& Davidson (1999), ao padrão específico do comportamento visual. O padrão de exploração visual 
limitado possibilita aos esquizofrênicos extrair informações pontuais suficientes para julgar as expressões emocionais principais, falhando na identificação e integração das informações sutis para uma percepção holística da face. Tal percepção é necessária para o julgamento adequado a respeito da graduação na intensidade das emoções expressas.

Segundo Sachs e colaboradores (2004), julgamento das emoções é um processo cognitivo complexo que, além da exploração visual ampla, requer recursos atencionais, vigilância para o estímulo observado e de memória. Resultados revelaram altas correlações entre julgamento das faces triste versus feliz com o funcionamento intelectual, memória verbal/não-verbal e capacidade de abstração/flexibilidade. Os autores concluíram que funcionamento cognitivo prejudicado afeta o julgamento da intensidade de emoções, embora a discriminação das emoções principais esteja preservada. Tais achados podem ser explicados pelo mau funcionamento dos lobos frontais e temporais, responsáveis pela integração das informações, e sistema límbico, responsável pelo processamento das emoções.

Gur e colaboradores (2002) registraram a ativação cerebral através da ressonância magnética funcional (fMRI) durante as tarefas de discriminação das emoções faciais. Os resultados encontrados mostraram uma diminuição na ativação do sistema límbico, principalmente da amígdala do lobo esquerdo e hipocampo bilateral. Amígdala está diretamente ligada ao reconhecimento das emoções, já o hipocampo está envolvido com os processos de memória e tem papel importante na hora de julgar e comparar as emoções com as experiências passadas.

O objetivo do presente trabalho foi comparar o padrão dos movimentos oculares durante a inspeção de faces com conteúdos emocionais e neutras por pacientes com esquizofrenia e sujeitos-controle. Os parâmetros dos movimentos oculares foram correlacionados com a sintomatologia e o nível de funcionamento cognitivo dos pacientes com esquizofrenia.

\section{Método}

\section{Participantes}

Participaram do estudo 20 sujeitos separados em dois grupos: grupo experimental (GE) e grupo-controle (GC). GE foi composto por 10 sujeitos (7 homens e 3 mulheres) com diagnóstico de esquizofrenia de acordo com o DSM-IV-R, idade média de 29,3 anos e média de 7,6 anos de escolarização. Todos os sujeitos de GE estavam em atendimento ambulatorial com tratamento medicamentoso com antipsicótico atípico Risperidona ${ }^{\circledR}$. GC foi composto por 10 sujeitos (7 homens e 3 mulheres) escolhidos entre os funcionários de uma empresa de Psico-USF, v. 12, n. 1, p. 95-102, jan./jun. 2007 limpeza e pareados em função de idade, escolaridade e nível de inteligência. Nenhum dos sujeitos-controle teve histórico psiquiátrico na família até $3^{\circ}$ grau. Critérios de exclusão foram: atraso mental, histórico de traumatismo ou doença associada com seqüelas nas funções cognitivas, histórico de transtorno esquizoafetivo, abuso de substâncias ou transtornos desenvolvidos na infância. A participação dos sujeitos foi voluntária e todos assinaram o Termo de Consentimento Livre e Esclarecido, previamente aprovado pelo Comitê de Ética da Universidade Presbiteriana Mackenzie.

\section{Instrumentos}

A Escala das Síndromes Positiva e Negativa (PANSS) foi usada para a avaliação da severidade dos sintomas positivos e negativos dos sujeitos com esquizofrenia. Para a avaliação foi usada a versão brasileira da escala original traduzida e validada pelo Vessoni (1993). A escala é composta por 30 itens divididos em 3 grupos: escala de sintomas positivos, negativos e geral. $\mathrm{O}$ tipo sintomatológico do sujeito é determinado de acordo com a pontuação em cada uma das escalas, cada item podendo receber pontuação de 1 (ausente) a 7 (extremamente grave). Caso três ou mais sintomas recebam pontuação maior ou igual a 4 na escala positiva e menos que três sintomas na escala negativa, a sintomatologia é do tipo Positiva. Caso três ou mais sintomas recebam pontuação maior ou igual a 4 na escala negativa e menos que três sintomas na escala positiva, a sintomatologia é do tipo Negativa. Caso três ou mais sintomas recebam pontuação maior ou igual a $4 \mathrm{em}$ ambas as escalas, a sintomatologia é do tipo Mista. Quando os critérios anteriores não se aplicam, é classificada como Nenbum Tipo. O nível intelectual geral (QI) foi avaliado com Teste de Matrizes Progressivas Raven.

Para a avaliação do desempenho do reconhecimento de emoções em expressões faciais foi utilizada a versão adaptada do teste computadorizado de identificação de emoções faciais, Penn Emotion Acuity Test (PEAT), desenvolvida por Gur, Erwin \& Gur (1992). PEAT é composto por fotos em preto-e-branco de faces humanas de ambos os sexos, retratando expressão com conteúdo emocional feliz, triste e neutra. Para o presente trabalho, foram selecionadas 18 fotos de 6 indivíduos de ambos os sexos que foram pareados em dois tipos de estímulos: estímulo composto por faces feliz-neutra e estímulo composto por faces triste-neutra. $\mathrm{O}$ posicionamento das faces com conteúdo emocional foi contrabalançado e a ordem de apresentação dos estímulos foi aleatorizada. Os estímulos foram apresentados no computador Pentium III, e o registro dos movimentos oculares feito com o equipamento EyeGaze ${ }^{\circledR}$ (LC 
Technologies Inc). A gravação de movimento ocular foi feita no olho dominante.

\section{Procedimentos}

A aplicação da escala PANSS e Raven foi realizada na entrevista inicial por um psiquiatra e dois psicólogos treinados.

A prova de identificação de emoções faciais foi realizada numa sala escura sem iluminação natural. $\mathrm{O}$ sujeito foi solicitado a sentar-se a uma distância de aproximadamente 40 centímetros da tela do computador e apoiar a cabeça em uma queixeira para a minimização dos movimentos. O equipamento foi calibrado com programa EyeGaze com base no reflexo pupilar, tamanho da pupila e curvatura da córnea. Após a calibração a prova foi iniciada.

Os parâmetros de movimento ocular registrados automaticamente durante a inspeção das faces foram: número de fixações $(\mathrm{NF})$, duração das fixações (DF) e comprimento dos movimentos sacádicos (CS).

\section{Resultados}

A fim de comparar o padrão dos movimentos oculares nas faces, foram conduzidos testes $T$ para amostras pareadas para: NF nas faces, DF e CS. A comparação da soma dos $\mathrm{NF}$ em todas as pranchas revelou diferença significativa $\left(t_{[18]}=1,859 ; p=0,079\right)$ entre os dois grupos, sendo que esquizofrênicos apresentaram menor NF $(M=268,60 ; D P=38,15)$ do que os sujeitoscontrole $(M=297,7 ; \mathrm{DP}=31,52)$. Embora não tenha sido observada diferença significativa entre os dois grupos, a DF tendeu a ser maior para os esquizofrênicos $(\mathrm{M}=394,83 \mathrm{~ms} ; \mathrm{DP}=71,62)$ do que para os controles $(\mathrm{M}=362,04 ; \quad \mathrm{DP}=68,88)$. De fato, foi observada correlação inversa entre o NF e DF ( $r=-0,607 ; p=0,005)$. Também não foi observada diferença significativa para DS. A Figura 1 ilustra os traçados dos movimentos oculares produzidos por um paciente esquizofrênico e seu controle durante a inspeção visual de um dos pares de fotos de faces humanas.
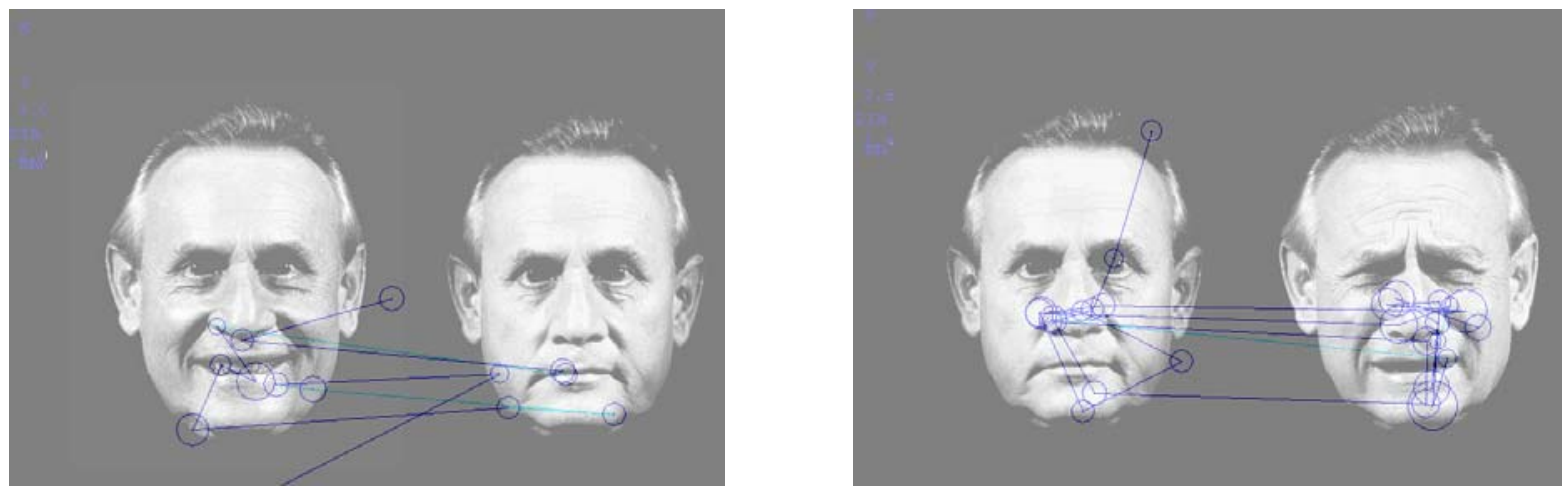

Figura 1 - Traçados dos movimentos oculares produzidos por um paciente esquizofrênico (esquerda) e seu controle (direita) durante a inspeção visual de um dos pares de fotos de faces humanas

Análises do padrão do tempo em que os sujeitos analisaram as faces foram conduzidas a fim de identificar preferência por uma das faces. ANOVA revelou efeito do tipo de face $\left(\mathrm{F}_{[2,36]}=16,455 ; p=0,000\right)$, sendo que tanto os controles quanto os esquizofrênicos olharam mais tempo para as faces com expressão, não sendo observada diferença significativa entre as faces tristes e felizes. Não foi observado efeito de interação entre condição e tipo de face. Os tempos médios para cada condição e tipo de estímulo estão descritos na Tabela 1.

Tabela 1 - Tempo médio (segundos) e desvio padrão para visualização das faces: neutra, feliz e triste por pacientes esquizofrênicos e sujeitos-controle

\begin{tabular}{lcccc}
\hline & Neutra & Feliz & Friste & Post hoc \\
\hline Esquizofrênicos & $3,975(0,819)$ & $4,711(0,747)$ & $4,759(0,935)$ & $\mathrm{a}, \mathrm{b}$, \\
Controle & $3,603(0,855)$ & $4,746(0,797)$ & $5,226(0,970)$ & $\mathrm{a}, \mathrm{b}$, \\
\hline
\end{tabular}

a) Neutra $x$ feliz; b) Neutra $x$ triste; c) Feliz $x$ triste 
A comparação de nível intelectual (QI) mostrou que, embora os controles $(M=29,40 ; D P=10,07)$ tenham obtido em média 3 pontos a mais que os esquizofrênicos $(\mathrm{M}=26,50 ; \mathrm{DP}=16,23)$, no escore total do Teste Raven, essa diferença não foi significativa.

A fim de observar as relações entre nível de inteligência e movimento ocular, correlações de Pearson entre pontuação no Raven e os parâmetros dos movimentos oculares foram conduzidas. Para os sujeitoscontrole não foi observada nenhuma correlação entre Raven e os parâmetros dos movimentos ocular. Já para os pacientes esquizofrênicos foi observada correlação positiva para TF tanto para as faces neutras quanto para as com expressão, tendo sido maior a correlação para aquelas com expressão de tristeza. Como não houve correlação do Raven com NF e TF, seriam esperadas correlações inversas com DS. De fato, foi observada correlação negativa entre a pontuação no Raven e DS, ou seja, esquizofrênicos com baixa pontuação no Raven apresentaram maiores dispersões na localização dos pontos de fixação. A Tabela 2 sumaria as correlações entre Raven e parâmetros dos movimentos oculares.

Tabela 2 - Correlação de Pearson e nível de significância entre a pontuação no Teste Matrizes Progressivas Raven e os parâmetros dos movimentos oculares para os sujeitos diagnosticados com esquizofrenia e controle

\begin{tabular}{lcccc}
\hline Parâmetros dos movimentos oculares & \multicolumn{2}{c}{ Esquizofrênicos } & \multicolumn{2}{c}{ Controle } \\
& Pearson & $\mathrm{p}$ & Pearson & $\mathrm{p}$ \\
\hline Tempo de fixação nas faces & 0,677 & 0,032 & $-0,054$ & 0,883 \\
Tempo de fixações nas faces (expressão) & 0,717 & 0,020 & 0,045 & 0,902 \\
Tempo de fixações nas faces alegre & 0,616 & 0,058 & 0,062 & 0,865 \\
Tempo de fixações nas faces triste & 0,711 & 0,021 & 0,022 & 0,953 \\
Número de fixações nas faces & 0,200 & 0,580 & $-0,014$ & 0,970 \\
Duração média das fixações & 0,475 & 0,165 & 0,038 & 0,918 \\
Distância média dos movimentos sacádicos & $-0,687$ & 0,028 & $-0,272$ & 0,447 \\
PANSS - negativa & $-0,518$ & 0,125 & & \\
PANSS - positiva & $-0,432$ & 0,212 & & \\
PANSS - geral & $-0,738$ & 0,015 & & \\
\hline
\end{tabular}

Análise de correlação de Pearson também revelou correlação inversa entre a pontuação do Raven e a pontuação na escala PANSS Geral. Assim, pacientes com maior número de sinais e sintomas para a esquizofrenia também obtiveram piores pontuações.

Em suma, como não foram observadas correlações entre inteligência e padrão de movimentos oculares nos sujeitos-controle e correlação inversa entre inteligência e escala PANSS para os esquizofrênicos, o padrão diferente dos movimentos oculares nos esquizofrênicos pode ser explicado a partir dos sinais e sintomas da doença.

Avaliação dos sintomas negativos e positivos pela escala PANSS não mostrou prevalência de nenhum tipo sintomatológico entre os sujeitos com esquizofrenia. Para cada uma das subescalas (positiva, negativa e geral) foi computada pontuação total, pontuação média e tipo sintomatológico. Os dados estão descritos na Tabela 3.

Tabela 3 - Pontuação e classificação dos pacientes esquizofrênicos na Escala das Síndromes Positiva e Negativa (PANSS)

\begin{tabular}{lccccccc}
\hline & \multicolumn{2}{c}{ Escala positiva } & \multicolumn{2}{c}{ Escala negativa } & \multicolumn{2}{c}{ Escala geral } & Tipo \\
Sujeitos & Pont. total & Pont. média & Pont. total & Pont. média & Pont. total & Pont. média & sintomatológico \\
\hline 1 & 9 & 1,29 & 8 & 1,14 & 18 & 1,13 & nenhum \\
2 & 16 & 2,29 & 15 & 2,14 & 45 & 2,81 & positivo \\
3 & 14 & 2 & 26 & 3,71 & 24 & 1,5 & negativo \\
4 & 17 & 2,43 & 13 & 1,86 & 28 & 1,75 & nenhum \\
5 & 13 & 1,86 & 14 & 2 & 25 & 1,56 & nenhum \\
6 & 19 & 2,71 & 26 & 3,71 & 37 & 2,31 & misto \\
7 & 12 & 1,71 & 7 & 1 & 21 & 1,31 & nenhum \\
8 & 17 & 2,43 & 24 & 3,43 & 33 & 2,06 & negativo \\
9 & 15 & 2,14 & 22 & 3,14 & 36 & 2,25 & nenhum \\
10 & 7 & 1 & 10 & 1,43 & 19 & 1,19 & nenhum \\
\hline
\end{tabular}

Psico-USF, v. 12, n. 1, p. 95-102, jan./jun. 2007 
A fim de verificar a correlação entre a sintomatologia dos quadros de esquizofrenia e preferência por faces, foram conduzidas correlações de Pearson. Resultados, descritos na Tabela 4, revelam correlação negativa entre sintomatologia negativa e tempo de fixação nas faces feliz e triste. Para a pontuação na PANSS Positivo, foi observada correlação negativa apenas para o tempo nas faces feliz. A pontuação na PANSS Geral se correlacionou positivamente com a distância média dos movimentos sacádicos. Dessa forma, pacientes com maiores incidências de sinais e sintomas tenderam a fazer fixações com distância média entre elas maior do que aqueles com baixa sintomatologia.

Tabela 4 - Correlação de Pearson e o nível de significância entre a pontuação na PANSS em razão da sintomatologia e dos parâmetros dos movimentos oculares para os sujeitos diagnosticados com esquizofrenia

\begin{tabular}{llccccc}
\hline \multirow{2}{*}{ Parâmetros dos movimentos oculares } & \multicolumn{2}{c}{ Escala negativa } & \multicolumn{2}{c}{$\begin{array}{c}\text { Escala } \\
\text { positiva }\end{array}$} & \multicolumn{2}{c}{$\begin{array}{c}\text { Escala } \\
\text { geral }\end{array}$} \\
& Pearson & $\mathrm{p}$ & Pearson & $\mathrm{p}$ & Pearson & $\mathrm{p}$ \\
\hline Tempo de fixação nas faces & $-0,508$ & 0,133 & $-0,362$ & 0,305 & $-0,342$ & 0,333 \\
Tempo de fixações nas faces (expressão) & $-0,754$ & 0,012 & $-0,430$ & 0,215 & $-0,339$ & 0,338 \\
Tempo de fixações nas faces feliz & $-0,787$ & 0,007 & $-0,623$ & 0,054 & $-0,409$ & 0,240 \\
Tempo de fixações nas faces triste & $-0,635$ & 0,049 & $-0,223$ & 0,536 & $-0,241$ & 0,503 \\
Número de fixações nas faces & 0,002 & 0,995 & $-0,039$ & 0,915 & $-0,040$ & 0,913 \\
Duração média das fixações & $-0,477$ & 0,163 & $-0,280$ & 0,433 & $-0,277$ & 0,438 \\
Distância média dos movimentos sacádicos & 0,404 & 0,247 & 0,384 & 0,273 & 0,571 & 0,085 \\
\hline
\end{tabular}

\section{Discussão}

O padrão de busca visual dos sujeitos esquizofrênicos encontrado no presente estudo é caracterizado por menor número de fixações, mas com duração maior. Esse padrão é semelhante ao padrão encontrado em outros estudos (Loughland, Williams \& Gordon, 2003, Loughland, Williams e Gordon, 2002, Ryu e cols., 2001), porém diverge destes em não encontrar diferença significativa no comprimento dos movimentos sacádicos.

O tempo de exploração das faces com conteúdo emocional foi maior para ambos os grupos. Embora o estudo do Loughland, Williams e Gordon (2002) aponte para diferenças nos padrões de movimentos oculares na exploração das faces com conteúdo emocional, os autores não descrevem o tempo de exploração. Isso pode ser em virtude da diferença no procedimento, pois no presente estudo as faces foram pareadas, uma com conteúdo emocional e outra neutra, diferentemente do estudo de Loughland e colaboradores (2002), em que o pareamento foi feito também entre as faces com conteúdo emocional. Por outro lado, Loughland e colaboradores (2002) encontraram variação no tempo de exploração nas faces que foram degradadas pelo computador. O tempo de exploração nessas faces peculiares foi maior do que em faces não degradadas. Podemos pressupor que as faces com conteúdo emocional, assim como as faces degradadas, são estímulos mais complexos do que as faces neutras e exigem, portanto, maior tempo de exploração.

A correlação entre as propriedades do movimento ocular e estado clínico dos sujeitos com esquizofrenia mostrou que quanto maior for a pontuação na escala de sintomas positivos e negativos menor é a duração das fixações nas faces. O efeito foi maior para sintomas negativos com faces triste e ausente para sintomas positivos com esse mesmo tipo de face. Além disso, a correlação positiva entre a escala geral e comprimento dos movimentos sacádicos mostra que estado clínico afeta o padrão de busca visual, junto com o tipo de estímulo apresentado. Nakayama e colaboradores (2003) descreveram mudanças no padrão de exploração visual dos pacientes na fase aguda e após 3 a 6 meses de tratamento. Com melhora no estado clínico o TF diminuiu e a DS aumentou. Nakayama e colaboradores (2003) encontraram correlação positiva entre os sintomas negativos e TF, achados encontrados também no presente estudo. A correlação negativa entre sintomas negativos e DS não foi observada no presente estudo, sendo que as diferenças nos estímulos e condição clínica dos participantes podem justificar tal diferença. Os estímulos usados pelo grupo de Nakayama foram desenhos pictográficos de rosto sorridente, de complexidade e tamanho inferior aos de fotos de faces humanas. Os sujeitos do presente estudo foram expostos a duas faces ao mesmo tempo com expressividade emocional diferente, aumentando assim ainda mais a 
complexidade do estímulo. Diante desse tipo de estímulo, sujeito esquizofrênico fora da fase aguda da doença apresenta padrão de busca com DS amplas com direções aleatórias, diferentemente do sujeito-controle cujas sacadas são direcionadas para as partes salientes da face (olhos, nariz e boca).

Alguns estudos com movimentos oculares levantam a questão sobre uma possível correlação entre padrão de movimento ocular e nível intelectual dos sujeitos (Sachs e cols., 2004). No presente estudo foi encontrada correlação positiva entre a pontuação no Raven e TF em todos os tipos de faces e correlação negativa entre Raven e DS. O dado importante reside no fato de que este padrão foi encontrado somente para o grupo dos esquizofrênicos, indicando uma correlação entre estado clínico e pontuação no Teste de Inteligência. O Teste de Matrizes Progressivas do Raven utilizado no presente estudo para a avaliação da inteligência é um teste visual de alta complexidade, pois contém um estímulo central e 8 estímulos periféricos. Quando levado em consideração o padrão diferenciado de exploração visual nos estímulos complexos como as faces humanas, podemos questionar a capacidade dos esquizofrênicos de fazer uma exploração visual eficiente nas pranchas do Teste Raven. O presente estudo indica que essa dificuldade fica mais evidente ao passo que aumenta a severidade dos sintomas clínicos. Estudos com amostra maior podem corroborar os achados obtidos no presente estudo e apontar maior validade estatística.

\section{Conclusão}

Este estudo pioneiro brasileiro descreveu as propriedades do movimento ocular durante a exploração das faces com conteúdo emocional. Mostrou as diferenças nas propriedades do movimento, correlacionando-as com estado clínico e nível intelectual. Apontou para a relevância dos estudos com movimentos oculares, uma vez que diversos instrumentos de avaliação psicológica comumente usados com pacientes esquizofrênicos demandam boa capacidade perceptual. Embora os resultados do presente estudo sejam compatíveis com a literatura estrangeira, novos estudos que comparem o efeito do tipo de medicação são necessários. A importância de tais estudos está no fato de se poder treinar as habilidades sociais dessa população e no diagnóstico precoce da doença, já que a busca visual da mesma população é característica.

\section{Refêrencias}

Calkins, M. E., Iacono, W. G. \& Curtis, C. E. (2003). Smooth pursuit and antisaccade performance evidence trait stability in schizophrenia patients and their relatives. International Journal of Psychophysiology, 49, 139-146.

Ettinger, U., Kumari, V., Crawford, T. J., Corr, P. J., Das, M., Zachariah, E., Hughes, C., Sumich, A. L., Rabe-Hesketh, E. \& Sharma, T. (2004). Smooth pursuit and antisaccade eye movements in siblings discordant foe schizophrenia. Journal of Psychiatric Research, 38, 177-184.

Goldman, R. S., Axelrod, B. N., Tndom, R., Ribeiro, S. C. M., Craig, K. \& Berent, S. (1993). Neuropsychological prediction of treatment efficacy on-year outcome in schizophrenia. Psychopathology, 126, 122-126.

Gur, R. C., Erwin, R. J. \& Gur, R. E. (1992). Neurobehavioral probes for physiologic neuroimaging studies. Archives of General Psychiatry, 49, 409-414.

Gur, R. E., McGrath, C., Chan, R. M., Schoroeder, L., Turner, T., Turetsky, B. I., Kohler, C., Alsop, D., Maldijian, J. \& Gur, R. C. (2002). An fMRI study of facial emotion processing in patients with schizophrenia. American Journal Psychiatry, 159(12), 1992-1999.

Holzman, P. S., Proctor, L. \& Huges, D. (1973). Eye tracking patterns in schizophrenia. Science, 181, 179181.

Holzman, P. S., Proctor, L. \& Huges, D. (1988). A single dominat gene can account for eye tracking dysfunctions and schizophrenia in offspring of discordant twins. Arch. Gen. Psychiatry, 45, 641-647.

Hori, Y., Fukuzako, H., Sugimoto, Y. \& Takigawa, M. (2002). Eye movement during the Rorschach Test in Schizophrenia. Psychiatry and Clinical Neuroschiences, 56, 409-418.

Javitt, D. C. \& Coyle, J. T. (2004). Decifrando a esquizofrenia. Scientific American Brasil, 21, 48- 55.

Kohler, C. G., Bilker, W., Haagendoorn, M. \& Gur, R. C. (2000). Emotion recognition deficit in schizophrenia: Association with symptomatology and cognition. Society of Biological Psychiatry, 48(2), 127-136.

Kojima, T., Matsushima, E., Ohta, K., Toru, M., Han, Y., Shen, Y., Moussaoui, D., David. I., Sato, K., Yamashita, I., Kathmann, N., Hippius, H., Thavundayil, J. X., Lal, S. Vasavan, N., Potkin, S. G. \& Prilipko, L. (2001). Stability of exploratory eye movements as a marker of schizophreni - a WHO multi-center study. Schizophrenia Research, 52, 203-213.

Loughland, C. M., Williams, L. M. \& Gordon, E. (2002). Visual scanpaths to positive and negative 
facial emotions in an outpatient schizophrenia sample. Schizophrenia Research, 55(1-2), 159-170.

Loughland, C. M., Williams, L. M. \& Gordon, E. (2003). Schizophrenia and affective disorder show different visual scanpath patterns to faces and facial expressions: Is this a trait versus state-based distinction? International Congress on Schizophrenia Research, 267.

Loughland, C. M., Williams, L. M., Harrys, A. W. (2004). Visual scanpath dysfunction in first-degree relatives of schizophrenia probands: Evidence for a vulnerability marker? Schizophrenia Research, 67(1), 1121.

Matsushima, E., Kojima, T. \& Ohta, K. (1998). Exploratory eye movement dysfunctions in patients with schizophrenia: Possibility as a discriminator for schizophrenia. Journal of Psychiatric Research, 32, 289295.

Nakamura, M., Matsushima, E., Ohta, K., Ando, K. \& Kojima, T. (2003). Relationship between attention and arousal level in schizophrenia. Psychiatry Clin. Neurosci., 57(5), 472-477.

Nakayama, H., Morita, K., Mori, K., Hirai, S. \& Maeda, H. (2003). Improvement of exploratory eye movements in schizophrenic patients during recovery period. Psychiatry and Clinical Neurosciences, 57, 169-176.

Obayashi, S., Matsushima, E., Ando, H. \& Kojima, T. (2003). Exploratory eye movements during the Benton Visual Retention Test: Characteristics of visual behavior in schizophrenia. Psychiatry and Clinical Neurosciences, 57, 409-415.

OMS (2000). The world health report 2000: Health System: improving performance. Genebra: OMS.

Rayner, K. (1998). Eye movements in reading and information processing: 20 years of research. Psychological Bulletin, 124, 372-422.

Ryu, H., Morita, Y., Shoji, Y. \& Maeda, H. (2001). Abnormal exploratory eye movements in schizophrenic patients vs healthy subjects. Acta Neurol. Scand., 104, 369-376.

Sachs, G., Steger-Wuchse, D., Kryspin-Exner, I., Gur, R. C. \& Katschnig, H. (2004) Facial recognition deficits and cognition in schizophrenia. Schizophrenia Research, 68 (1), 27-35.

Vessoni, A. L. N. (1993). Adaptação e estudo da confiabilidade da Escala de Avaliação das Síndromes Positiva e Negativa para a Esquizofrenia no Brasil. (Dissertação de Mestrado). São Paulo: Escola Paulista de Medicina.

Williams, L. M., Loughland, C. M., Gordon, E. \& Davidson, D. (1999). Visual scanpaths in schizophrenia: Is there a deficit in face recognition? Schizophrenia Research, 40 (3), 189-199.

Recebido em fevereiro de 2006 Aprovado em março de 2007

Sobre os autores:

Katerina Lukasova é psicóloga e mestre em distúrbios do desenvolvimento pela Universidade Presbiteriana Mackenzie.

Elizeu Coutinho de Macedo é psicólogo, mestre e doutor em Psicologia Experimental pela Universidade de São Paulo e atua como professor do Programa de Pós-Graduação em Distúrbios do Desenvolvimento da Universidade Presbiteriana Mackenzie.

Marcel Chucre Valois é psicólogo clínico com atuação em Centro de Apoio Psicossocial (Caps-adulto) do município de Carapicuíba-SP.

Gamaliel Coutinho de Macedo é médico psiquiatra com atuação clínica e colaborador do projeto de esquizofrenia (PROJESQ-IPQ-HC-FMUSP).

José Salomão Schwartzman é médico e doutor em Neurologia pela Universidade Federal de São Paulo, professor titular na Pós-Graduação em Distúrbios do Desenvolvimento da Universidade Presbiteriana Mackenzie e editor científico do periódico Temas Sobre Desenvolvimento. 